\title{
GASDYNAMICS AND STARBURSTS IN INTERACTING GALAXIES
}

\author{
J. CHRISTOPHER MIHOS \\ Department of Astronomy \\ Case Western Reserve University
}

\begin{abstract}
The onset of gaseous inflows and central activity in interacting galaxies is driven largely by induced bars in the host galaxies. The stability of galaxies against growing bar modes is a direct function of their structural properties - galaxies with central bulges or low disk surface densities are more stable against central starbursts than are bulgeless or disk-dominated systems. Low surface brightness galaxies prove less prone to bar formation and central starbursts than do normal high surface brightness galaxies. This stability of LSB disks also resolves many of the dynamical pitfalls encountered when attempting to link poststarburst " $\mathrm{E}+\mathrm{A}$ " galaxies to interactions involving normal high surface brightness galaxy progenitors.
\end{abstract}

\section{Introduction}

Overwhelming evidence indicates that galactic collisions can lead to a large scale redistribution of gas in galaxies, driving strong nuclear inflows and fueling central activity (starburst and/or AGN) in many interacting systems. However, a one-to-one correlation between interactions and central starbursts is not evident - many interacting systems show only modest star forming activity, distributed throughout the body of the galaxy. What, then, determines the gasdynamical and star forming response of a galaxy to a gravitational encounter? Detailed N-body simulations of interacting systems have shown that the onset of gaseous inflows is intimately tied to the formation of global bars, which act to drive gas inwards to the central regions. As such, the question of induced star formation becomes one of induced bar formation - that is, the onset of inflow and activity is determined by a galaxy's stability against growing bar modes. 
I describe how the structural properties of galaxies can influence the gasdynamical and star-forming response of galaxies to an interaction. I focus first on major mergers and the effects of central bulges, then turn to more subtle "flyby" encounters and the role of disk surface density in driving starburst activity. We find that differences in galaxy structure lead to significantly different responses; much of the variance in the properties of interacting systems can be traced to differences in the progenitor galaxies.

\section{Gasdynamics in Major Mergers}

Major mergers of equal mass disk galaxies are thought to result in the most dramatic starburst events. The "ultraluminous" infrared galaxies (ULIRGs) are prime examples of this process, where $\sim 10^{12} M_{\odot}$ of gas has been driven into their central regions, fueling intense $\left(L_{\mathrm{IR}}>10^{12} L_{\odot}\right)$ activity (see, e.g., Sanders \& Mirabel 1996). To study the evolution of such mergers, we employ N-body models to follow the combined gravitational, hydrodynamic, and star-forming evolution of galaxies experiencing a merging event (Mihos \& Hernquist 1994ab; 1996).

We contrast models in which the merging galaxies have different structural properties - in particular, galaxy models with and without central bulges. We employ a system of units wherein the disk mass $M_{\mathrm{d}}=1$, the scale length of the disk $h=1$, and the gravitational constant $G=1$. In both models, the galaxies consist of an exponential disk of stars and gas $\left(M_{\text {gas }}=0.1\right)$ embedded in a spherical dark matter halo with mass $M_{\mathrm{h}}=5.8$ and core radius $\gamma=1$, truncated at $r=10 h$. In the model which includes a central bulge, the bulge possesses a Hernquist (1990) profile, with mass $M_{\mathrm{b}}=1 / 3$ and scale length $a=0.2$. Rotation curves for the different models are shown in Figure 1ab. Star formation is included via a simple Schmidt law: SFR $\sim \rho_{\text {gas }}^{1.5}$ (see Mihos \& Hernquist 1994b). The galaxies are placed on (initially) parabolic orbits, with a Keplerian pericenter of $R_{\mathrm{p}}=2.5$. One disk is exactly prograde, the other is inclined by $71^{\circ}$ to the orbital plane. Figure 2 shows the inflow and star forming properties of each model; images of the models can be found in Mihos \& Hernquist (1994a, 1996).

Even though the interaction parameters are identical, the star forming response of the two models is dramatically different. The galaxies without bulges rapidly develop strong bars - the $m=2$ mode in the stellar disk dominates the mass distribution shortly after the galaxies first collide. Gas is compressed along this bar, forming a gaseous bar which slightly leads the stellar bar. This offset between the stellar and gaseous bars results in a net torque on the gas, driving the strong inflow of gas into the nuclear regions. At this time, starburst activity is triggered in each nucleus while the galaxies are still widely separated. These starbursts deplete the gas, so 


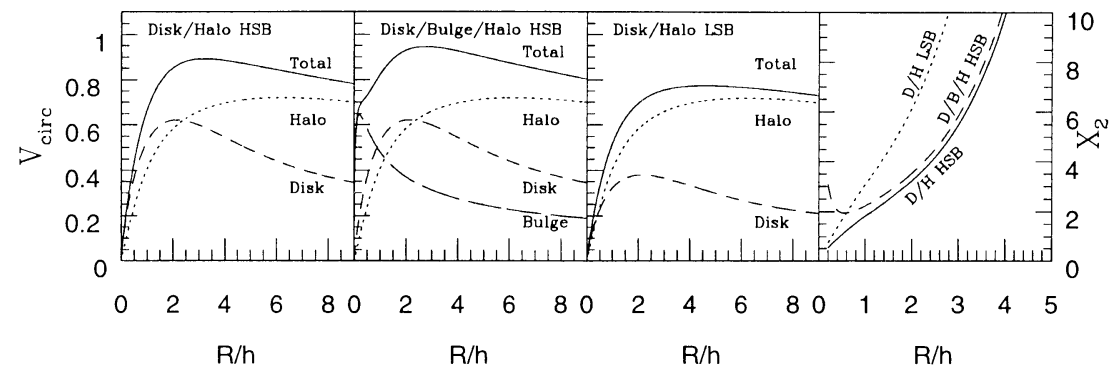

Figure 1. Rotation curves of galaxy models. The first three panels show the contribution of different components to the total rotation curve of each galaxy, while the final panel shows the Toomre $X_{2}$ bar stability parameter for each model.

that when the galaxies ultimately merge, they are gas poor and lack the fuel to power any strong starburst associated with the final merging. As such, these models are poor representations of ULIRGs, which are gas-rich, late stage mergers with strong central activity. Evidently a major merger alone is not a sufficient condition to trigger ultraluminous activity; some other criteria is necessary.

In contrast, the merger involving galaxies with bulges has a very different history of inflow and starburst activity. The presence of a central bulge acts to stabilize the galactic disks against the growth of bar instabilities; instead, the galaxies form tightly wound spiral arms which provide a weaker torque on the disk gas. As a result, the gas inflow occurs in two stages. Initially, the gas moves inwards, but "hangs up" at a radius of a few $\mathrm{kpc}$, where the bulge dominates the mass distribution and the disk torques are weaker. This weak inflow results in only a modest enhancement of the star formation rate, and the gas is not strongly depleted. When the galaxies do finally merge, the accompanying strong torques result in a second phase of inflow - the gas in both galaxies is very quickly driven into the center of the merger, and an extreme starburst event is triggered. Unlike the bulgeless merger, this merger with bulges has properties (morphology, gas content, starburst strength) which compare favorably with observed ultraluminous infrared galaxies. It is the internal dynamics of the merging galaxies which is the necessary criterion for the formation of ULIRGs.

As these models demonstrate, the detailed response of galaxies to a merger depends critically on their stability against the onset of global bar modes. This stability has been characterized by the Toomre $X_{2}$ parame-

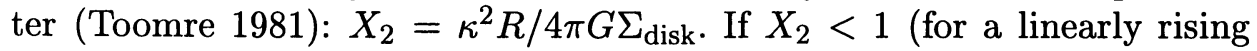
rotation curve) or $X_{2}<3$ (for a flat rotation curve), disks are susceptible to growing $m=2$ modes. Fig $1 \mathrm{~d}$ shows $X_{2}$ for the different models - by 

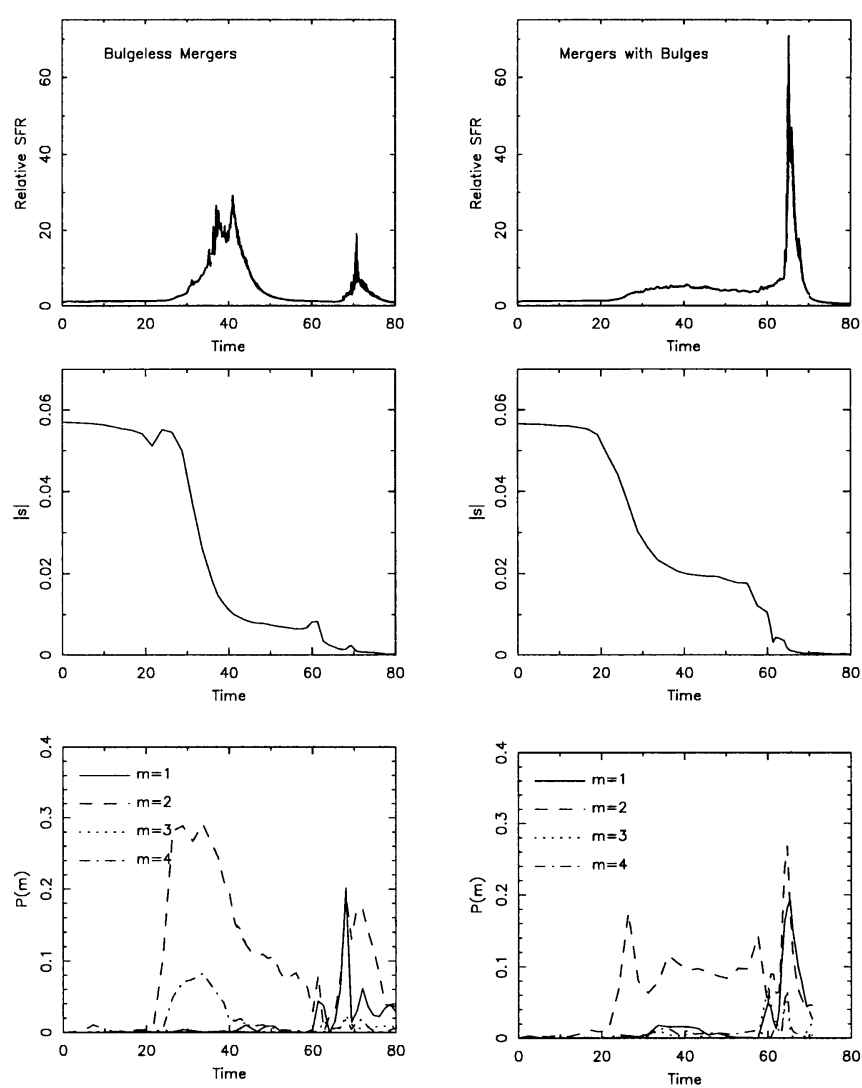

Figure 2. Gas inflow and starburst activity in major mergers. Left panels show the evolution of the bulgeless galaxy models, while the right panels show the evolution of the models with central bulges. Top panels show the star formation history in the models (assuming a Schmidt law for star formation); middle panels show the spin angular momentum of the inflowing gas in the prograde galaxy; and bottom panels show a Fourier decomposition of the stellar mass distribution in the prograde disk. The rotation period at the half mass radius is $T_{\text {rot }} \sim 15$. Initial collision occurs at $T=24$; the final merging occurs at $T=65-70$.

changing the shape of the rotation curve, the bulge acts to stabilize the inner disk against bar modes. Without bulges, colliding disk galaxies become bar unstable on a dynamical timescale, and experience early inflows and central activity. Adding a bulge inhibits bar formation and the associated early inflow, resulting in more dramatic activity when the galaxies ultimately merge. Clearly these two model represent "endpoints" of a distribution of bulge:disk ratios in galaxies; the response of individual systems will depend in detail on the their structural properties and progenitor type. 


\section{Flyby Encounters and LSBs}

The previous merger models show the connection between inflow, starbursts, and disk stability. However, there is another path to disk stability besides central bulges, and that is through lowered disk surface density. If the density of the disk is sufficiently low (at fixed rotation velocity), perturbations cannot be amplified into strong bar modes, and bar-induced inflows are suppressed. Such may be the case in low surface brightness (LSB) disk galaxies, which have low disk surface densities and large dark matter contents (de Blok \& McGaugh 1996, 1997).

To examine how disk surface density influences inflow and star forming activity during galaxy interactions, we look at the evolution of galaxies experiencing an equal-mass, non-merging "flyby" encounters. Again, two models are contrasted. The first, representing a high surface brightness (HSB) disk galaxy, is the bulgeless disk/halo galaxy model employed in the previous merger simulations. The second model, representing a LSB disk galaxy, is simply the HSB model with the disk surface density reduced by a factor of 2.5 - i.e., $\Delta \mu_{0} \sim 1 \mathrm{mag} / \operatorname{arcsec}^{2}$ for a similar $(M / L)_{*}$. The rotation curve for this model is shown in Figure 1; the low disk surface density results in a greater stability against growing disk modes than in HSB disk galaxies (as shown by the higher value of $X_{2}$ ), save for the very central regions where the disk still contributes an appreciable amount of the total mass density. Finally, compared to HSBs, LSBs generally have a higher gas-to-baryonic mass ratio and flatter gas mass profiles (de Blok et al. 1996; McGaugh \& de Blok 1997); this is reflected in the LSB model which possesses a flatter gas mass profile with $M_{\text {gas }} / M_{\text {disk }}=1 / 3$ (see Figure $3)$.

The flyby interactions involve parabolic orbits with pericenter separation of $R_{\mathrm{p}}=10 \mathrm{~h}$. Figure 3 shows the evolution of the ISM component in the different models. In the prograde HSB encounter, the galaxy quickly develops a strong bar (see Mihos et al. 1997); gas is compressed along this bar and is rapidly driven inwards. By $T=36$, only one half-mass rotation period for the disk, already $\sim 30 \%$ of the gas has been driven into the inner kpc (assuming a Milky Way scaling for the model); the calculation was stopped here, but inflow continues along the strong bar in the model.

By contrast, the prograde LSB disk lacks sufficient self-gravity in the disk to amplify the perturbation of the interaction into a strong bar. Instead, the galaxy develops a milder oval distortion with strong spiral arms. Gas is compressed along these arms, and fragments into small clumps throughout the disk; presumably these would be sites of enhanced star formation. There is some mild inwards migration of gas in the system, but the mass distribution in the inner scale length is largely unchanged - without 


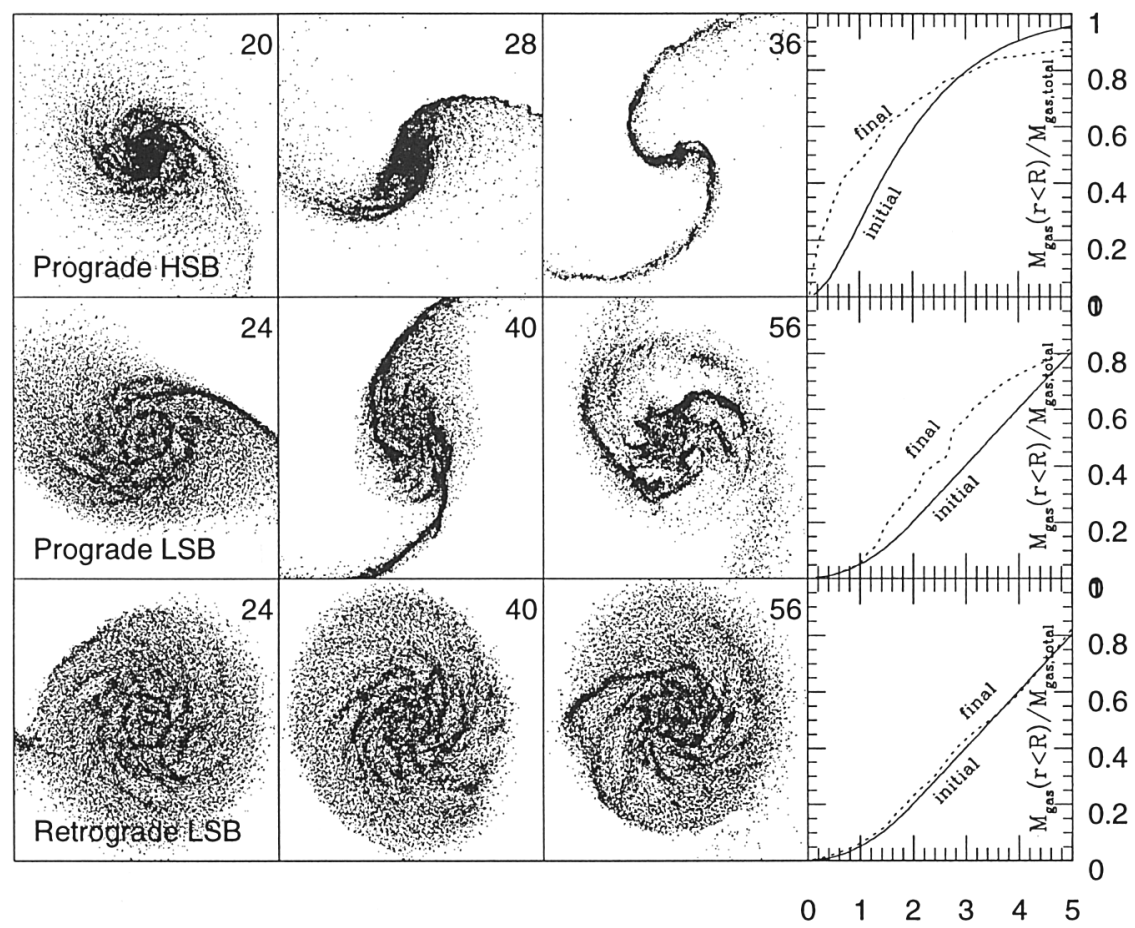

$\mathrm{R} / \mathrm{h}$

Figure 3. The evolution of the ISM in flyby interactions. In each strip, the first three panels show the morphology of the disk gas after the galaxies pass closest approach (at $T=24)$; the final panel compares the initial and final $(T=36$ for the HSB disk, $T=56$ for the LSB disks) cumulative gas mass profiles in each model. The rotation period at the half-mass radius for the disk is $T_{\text {rot }} \sim 15$. Note the different timescales between the HSB and LSB models; the HSB model quickly develops a bar which drives rapid inflow. The LSB models evolve much more slowly.

a strong bar, little inflow into the central regions occurs. We emphasize that the prograde geometry is the "worst case" scenario for driving instabilities in disks, due to the resonance between orbital and rotational motion; other geometries will be much less damaging. For example, the last set of panels in Figure 3 shows a retrograde LSB encounter - very little evolution is observed, even during this relatively close encounter.

Clearly the low disk surface density and high dark matter content of LSB disks affords them a great deal of stability against bars and induced gas inflows. This stability actually strengthens with declining surface brightness (as surface density decreases and dark matter becomes ever more dominant; de Blok \& McGaugh 1997), such that very low surface brightness galaxies 
may in fact be very stable systems, not easily destroyed or turned into starburst HII galaxies by casual interactions. Instead, mild evolution in surface brightness may result due to enhanced disk star formation.

Finally, we emphasize that the stability described here pertains to the internal amplification of perturbations into bar modes. This discussion is most apropos to mild interactions or secular evolution of LSBs. Mergers or continual interactions (such as in a cluster environment) may act to overwhelm the disk stability and drive additional activity even in these dark matter dominated systems.

\section{The Progenitors of $\mathbf{E}+\mathbf{A s}$}

"E+A" galaxies are systems with spectral characteristics identifying them as having experienced a strong starburst in the past $10^{9}$ years, after which star formation ceased entirely. The fact that such systems are observed in both the cluster and field environments (Zabludoff et al. 1996) argues in favor of formation mechanisms which are not cluster specific, such as galaxy interactions and mergers (e.g., Lavery et al. 1992). While this picture of mergers driving the formation of strong E+A systems works on a qualitative basis, upon closer scrutiny several inconsistencies appear when trying to invoke "normal" (i.e., HSB) spiral galaxies as the merging progenitors. Alternatively, I argue that LSB progenitors may solve a number of these inconsistencies. To wit:

1. High starburst mass fractions: Extreme E+A galaxies may have starburst mass fractions $M_{\text {burst }} / M_{*} \gtrsim 0.2$ (Couch \& Sharples 1987; Barger et al. 1996; Liu \& Green 1996). Such high burst masses indicate the galaxies must have been extremely gas-rich; Milky Way-type spirals simply lack sufficient gas to fuel such a starburst. LSBs, on the other hand, are the most gas-rich objects in the local universe (McGaugh \& de Blok 1997).

2. Spatially extended burst populations: In E+As the young stellar population is often spatially extended, and not confined to the nuclear region (e.g., Franx 1993; Caldwell et al. 1996). This is contrary to observations of local starburst galaxies, which are predominantly nuclear starbursts (e.g., M82, or the ULIRGs). However, the stability of LSB galaxies results in a global response to interactions, as gas fragments throughout the disk but is not driven into the galaxies' centers. Disk star formation, rather than nuclear starbursts, is the likely outcome.

3. E+As in galaxy pairs: The fact that some $\mathrm{E}+\mathrm{As}$ are found in interacting pairs (Zabludoff 1996; Wirth 1996) raises a timescale problem - if the interaction caused the starburst, why is the system a post-starburst system, even though the interaction is still ongoing? Some mechanism 
must act to shut off star formation, independent of the interaction phase. The low gas densities of LSBs, coupled with a threshold density for star formation (e.g., Kennicutt 1989, van der Hulst et al. 1993), may provide such a shutoff mechanism. If the initial collision drives disk gas above threshold, strong disk star formation ensues. As this star formation depletes the gas, the gas density drops back below threshold, and star formation is stopped. Because the cessation of star formation is linked to local dynamical conditions, the starburst can terminate irrespective of the dynamical phase of the interaction.

4. Disky E+As: Some E+A galaxies are disky systems (Caldwell et al. 1996; Wirth 1996; Franx, this volume). Such E+As can't form through major mergers, which destroy galactic disks. If interactions drive the formation of disky E+As, they must involve low mass accretions or flyby passages. With starburst efficiencies lower in these types of interactions, the gas reservoir must be extremely large, as found in LSBs.

Certainly not all $\mathrm{E}+$ As need arise from interacting LSB disks - many $\mathrm{E}+$ As show clear merger morphologies, or possess weaker burst strengths. However, the interdependence described here between galactic structure, disk stability, gas inflows, and starbursts suggests that the variety of progenitor galaxies available in the Universe necessarily dictates a variety of poststarburst $\mathrm{E}+\mathrm{A}$ galaxies. The $\mathrm{E}+\mathrm{As}$ which do not easily fit into the picture of interactions involving normal "Hubble-type" spirals may in fact follow from LSB progenitors.

\section{References}

Barger, A.J. et al. 1996, MNRAS, 279, 1

Caldwell, N. et al. 1996, AJ, 111, 78

Couch, W.J., \& Sharples, R.M. 1987, MNRAS, 229, 423

de Blok, W.J.G., \& McGaugh, S.S. 1996, ApJ, 469, L89

de Blok, W.J.G., \& McGaugh, S.S. 1997, MNRAS, 290, 533

de Blok, W.J.G., McGaugh, S.S., \& van der Hulst, J.M. 1996, MNRAS, 283, 18

Franx, M. 1993, ApJ, 407, L5

Lavery, R.J. et al. 1992, AJ, 104, 2067

Liu, C.T., \& Green, R.F. 1996, ApJ, 458, L63

McGaugh, S.S., \& de Blok, W.J.G. 1997, ApJ, 481, 689

Mihos, J.C., \& Hernquist, L. 1994a, ApJ, 431, L9

Mihos, J.C., \& Hernquist, L. 1994b, ApJ, 437, 611

Mihos, J.C., \& Hernquist, L. 1996, ApJ, 464, 641

Mihos, J.C., McGaugh, S.S., \& de Blok, W.J.G. 1997, ApJ, 477, L79

Kennicutt, R. 1989, ApJ, 344, 685

Sanders, D.B., \& Mirabel, I.F. 1996, ARAA, 34, 749

Toomre, A. 1981, in The Structure and Evolution of Normal Galaxies, eds. S.M. Fall \&

D. Lynden-Bell (Cambridge: Cambridge University Press), 111

van der Hulst, J.M. et al. 1993, AJ, 106, 548

Wirth, G.D. 1996, Ph.D. thesis, UC Santa Cruz

Zabludoff, A. et al. 1996, ApJ, 466, 106 